\title{
Direct longitudinal laser acceleration of electrons in free space
}

\author{
Sergio Carbajo, ${ }^{1,2,3,{ }^{*}}$ Emilio A. Nanni, ${ }^{1}$ Liang Jie Wong, ${ }^{1,4}$ Gustavo Moriena, ${ }^{5}$ Phillip \\ D. Keathley, ${ }^{1}$ Guillaume Laurent, ${ }^{1}$ R. J. Dwayne Miller, ${ }^{3,5}$ and Franz X. Kärtner ${ }^{1,2,3}$ \\ ${ }^{1}$ Department of Electrical Engineering and Computer Science, and Research Laboratory of Electronics, \\ Massachusetts Institute of Technology, 77 Massachusetts Avenue, Cambridge, Massachusetts 02139, USA \\ ${ }^{2}$ Center for Free-Electron Laser Science and The Hamburg Center for Ultrafast Imaging, \\ Luruper Chaussee 149, 22607 Hamburg, Germany \\ ${ }^{3}$ Deutsches Elektronen Synchrotron, and Department of Physics, \\ University of Hamburg, Notkestraße 85, Hamburg 22761, Germany \\ ${ }^{4}$ Singapore Institute of Manufacturing Technology, \\ 71 Nanyang Drive, Singapore 638075, Republic of Singapore \\ ${ }^{5}$ Max Planck Institute for the Structure and Dynamics of Matter, Luruper Chaussee 149, 22761 Hamburg, \\ Germany and the Department of Chemistry and Physics, University of Toronto, 80 Saint George Street, \\ Toronto, Ontario M5S 3H4, Canada
}

(Received 2 July 2015; published 24 February 2016)

Compact laser-driven accelerators are pursued heavily worldwide because they make novel methods and tools invented at national laboratories widely accessible in science, health, security, and technology [V. Malka et al., Principles and applications of compact laser-plasma accelerators, Nat. Phys. 4, 447 (2008)]. Current leading laser-based accelerator technologies [S. P. D. Mangles et al., Monoenergetic beams of relativistic electrons from intense laser-plasma interactions, Nature (London) 431, 535 (2004); T. Toncian et al., Ultrafast laser-driven microlens to focus and energy-select mega-electron volt protons, Science 312, 410 (2006); S. Tokita et al. Single-shot ultrafast electron diffraction with a laser-accelerated sub-MeV electron pulse, Appl. Phys. Lett. 95, 111911 (2009)] rely on a medium to assist the light to particle energy transfer. The medium imposes material limitations or may introduce inhomogeneous fields [J. R. Dwyer et al., Femtosecond electron diffraction: "Making the molecular movie,", Phil. Trans. R. Soc. A 364, 741 (2006)]. The advent of few cycle ultraintense radially polarized lasers [S. Carbajo et al., Efficient generation of ultraintense few-cycle radially polarized laser pulses, Opt. Lett. 39, 2487 (2014)] has ushered in a novel accelerator concept [L. J. Wong and F. X. Kärtner, Direct acceleration of an electron in infinite vacuum by a pulsed radially polarized laser beam, Opt. Express 18, 25035 (2010); F. Pierre-Louis et al. Direct-field electron acceleration with ultrafast radially polarized laser beams: Scaling laws and optimization, J. Phys. B 43, 025401 (2010); Y. I. Salamin, Electron acceleration from rest in vacuum by an axicon Gaussian laser beam, Phys. Rev. A 73, 043402 (2006); C. Varin and M. Piché, Relativistic attosecond electron pulses from a freespace laser-acceleration scheme, Phys. Rev. E 74, 045602 (2006); A. Sell and F. X. Kärtner, Attosecond electron bunches accelerated and compressed by radially polarized laser pulses and soft-x-ray pulses from optical undulators, J. Phys. B 47, 015601 (2014)] avoiding the need of a medium or guiding structure entirely to achieve strong longitudinal energy transfer. Here we present the first observation of direct longitudinal laser acceleration of nonrelativistic electrons that undergo highly directional multi-GeV/m accelerating gradients. This demonstration opens a new frontier for direct laser-driven particle acceleration capable of creating well collimated and relativistic attosecond electron bunches [C. Varin and M. Piché, Relativistic attosecond electron pulses from a free-space laser-acceleration scheme, Phys. Rev. E 74, 045602 (2006)] and x-ray pulses [A. Sell and F. X. Kärtner, Attosecond electron bunches accelerated and compressed by radially polarized laser pulses and soft-x-ray pulses from optical undulators, J. Phys. B 47, 015601 (2014)].

DOI: 10.1103/PhysRevAccelBeams.19.021303

\footnotetext{
* Corresponding author. scarbajo@stanford.edu
}

Published by the American Physical Society under the terms of the Creative Commons Attribution 3.0 License. Further distribution of this work must maintain attribution to the author $(s)$ and the published article's title, journal citation, and DOI.

\section{INTRODUCTION}

High-energy few-cycle laser sources with cylindrical vector beams remain relatively unexplored today, which has traditionally limited the generation of laser pulses with relativistic intensities to linearly polarized lasers. Radially polarized beams - a type of cylindrical vector beam - are specially of interest to relativistic laser-particle interactions 


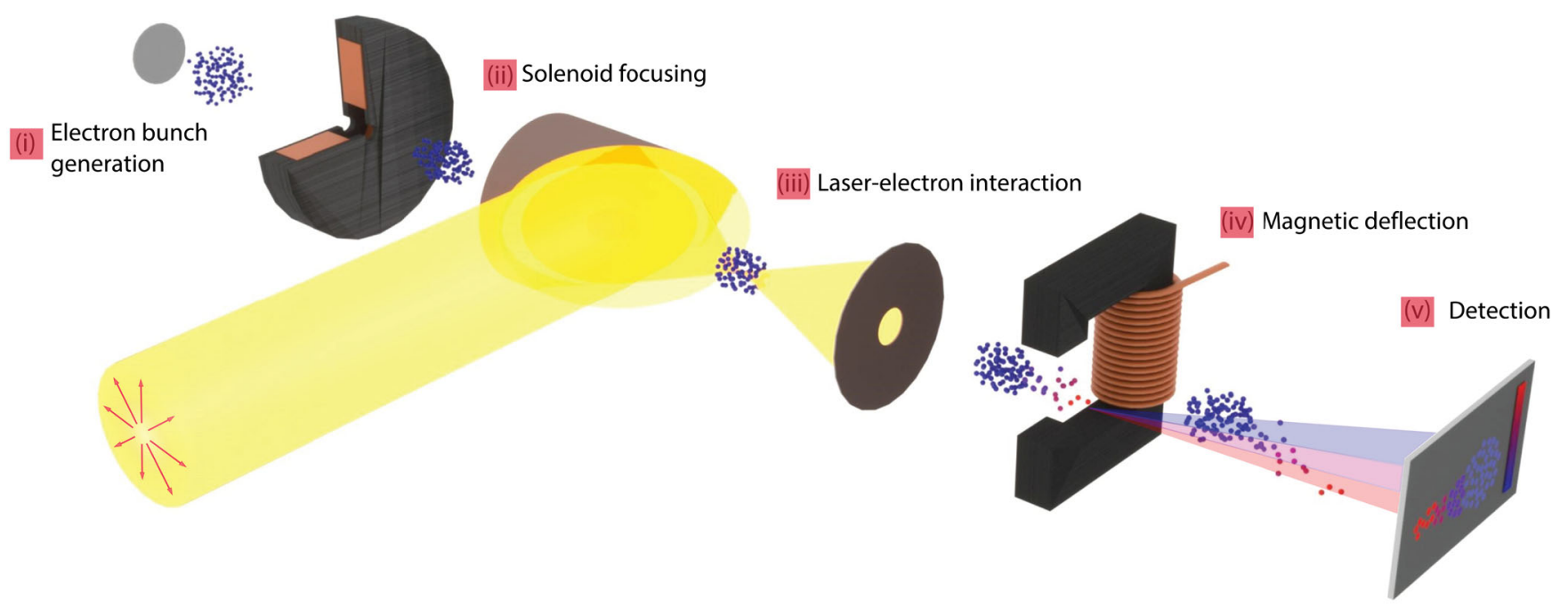

FIG. 1. Conceptual schematic. A nonrelativistic monoenergetic electron bunch is generated via a $40 \mathrm{kV}$ dc gun (i) triggered by a small fraction of the optical beam. The electron bunch is then focused (ii) at the laser-electron interaction point using a solenoid. A subsequent off-axis parabola with a thru-hole allows for tight focusing of the optical beam to overlap with the electron bunch (iii). After the interaction, the electrons enter a dipole magnet deflector (iv) and are then mapped onto a CCD camera from the emission of a fluorescent screen placed after a microchannel plate detector $(\mathrm{v})$.

because they exhibit cylindrical symmetry of the fieldvector distribution and can therefore be focused much more tightly, down to about 0.6 times the cross-sectional area of the diffraction-limited foci of linearly polarized beams $[12,13]$. As a consequence, the threshold power required to reach relativistic intensity levels becomes significantly less demanding. Moreover, the longitudinal field components of a focused radially polarized beam are uniquely enhanced, with approximately twice the field strength of beams with any other polarization, thereby making it the ideal driver for direct-field on-axis particle acceleration in vacuum $[7,8,10,14,15]$.

Despite these unique properties and their promising theoretical performance in direct particle acceleration, radially polarized pulses have only been used for acceleration of electrons in low density gas chambers and are consequently inconclusive in revealing direct particle acceleration strictly in vacuum [16,17]. Until recently, methods to generate radially polarized beams [18-20] have faced shortcomings to scale up and deliver the minimum intensity requirements to access the relativistic regime of laser-electron interaction, well above $10^{19} \mathrm{~W} / \mathrm{cm}^{2}$ in the case of optical pulses. In fact, this technological void has given way to other laser acceleration schemes [21-27] capable of yielding multi-GeV/m accelerating gradients and reaching well into the relativistic electron energy regime. However, these schemes require the presence of a medium for net energy transfer, which may translate into material breakdown or wave-breaking regime limitations for instance in the case of inverse Cherenkov acceleration [23] and plasma wakefield acceleration [28], respectively, or still face several challenges-large emittance, energy spread, and divergence [28,29] among others-which can severely hamper their use in practical applications. The potential of free-space acceleration is vast. For instance, previous work [7] has predicted that petawatt peak-power lasers could accelerate electrons to above $\mathrm{GeV}$ energies directly from rest. Direct vacuum acceleration employing radially polarized laser pulses is inherently a purely freespace scheme capable of creating well-collimated and relativistic attosecond electron bunches [10,11] and it is thereby unrestrained from the material limitations found in other methods. In spite of such great potential, the question of free-space acceleration has remained unanswered primarily due to a not yet mature drive source technology.

Here we show the first experimental demonstration under more modest conditions that serves as a proof of principle for direct (i.e., in vacuum) longitudinal (i.e., in the forward direction along the optical axis) laser acceleration of electrons, which is driven exclusively by the electric field formed by the radially polarized electromagnetic pulse at focus. This method takes advantage of the strong longitudinal electric field at beam center, where the transverse field components are not as strong, to accelerate electrons along the optical axis without additional mediation. The key enabling technology relies on a recently demonstrated efficient scheme to generate highly focusable intense few-cycle radially polarized laser pulses [6]. Such pulses are produced by combining a gas-filled hollow-waveguide compressor and a broadband linear-toradial polarization converter with an ultrafast Ti:sapphire chirped pulse amplifier. The polarization conversion is based on a segmented wave plate, which can be manufactured to withhold substantially larger energies through large apertures. The system delivers three-cycle carrierenvelope-phase stable radially polarized laser pulses at 
(a)

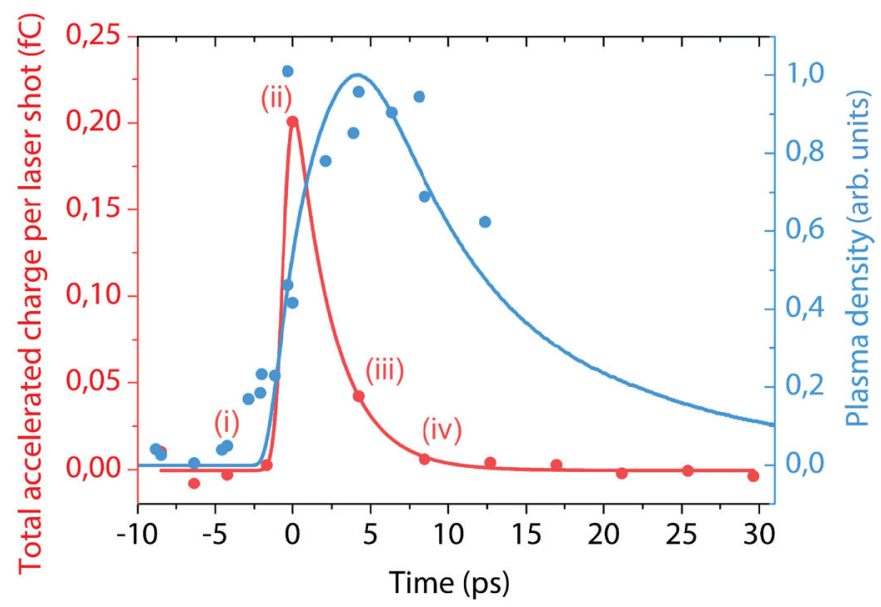

(c)
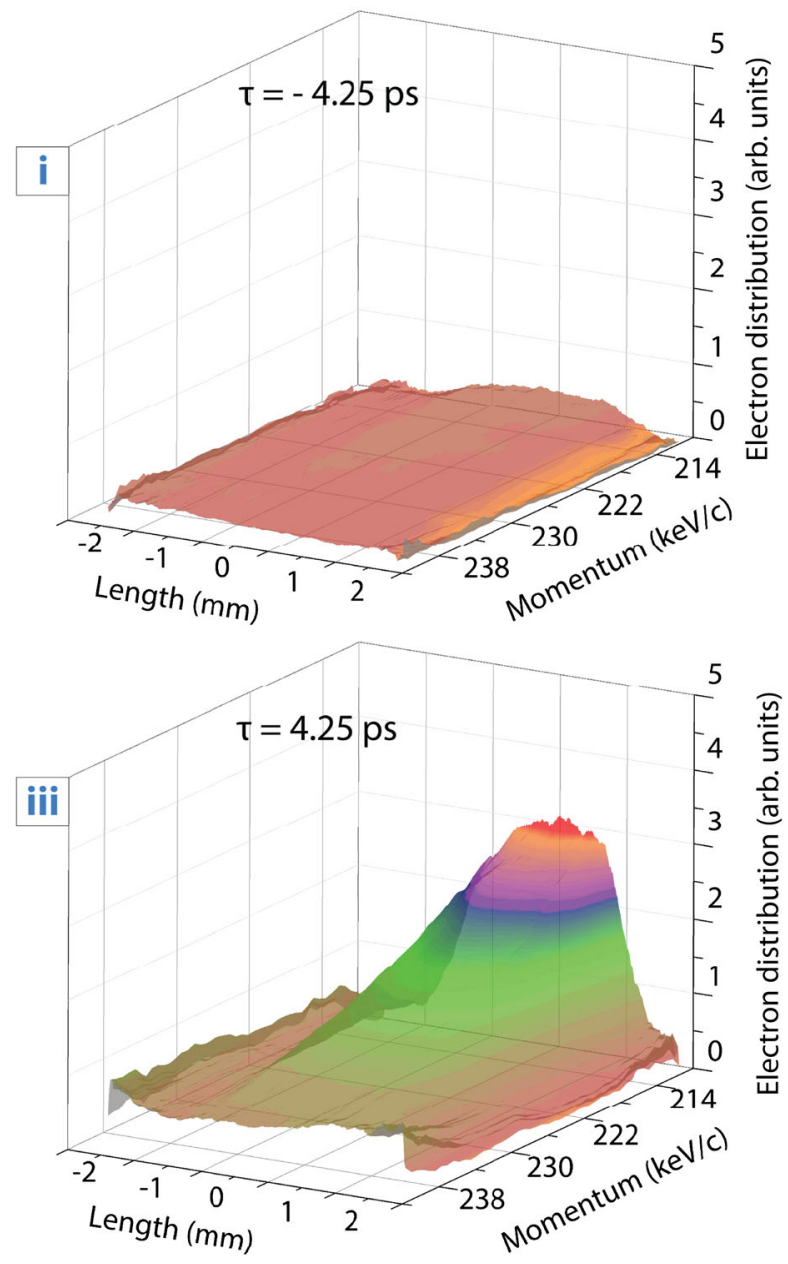

(b)

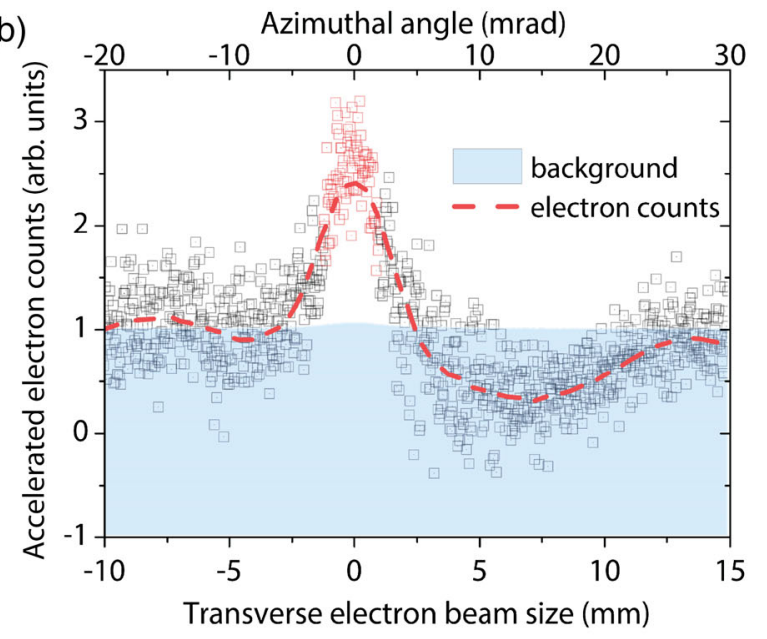

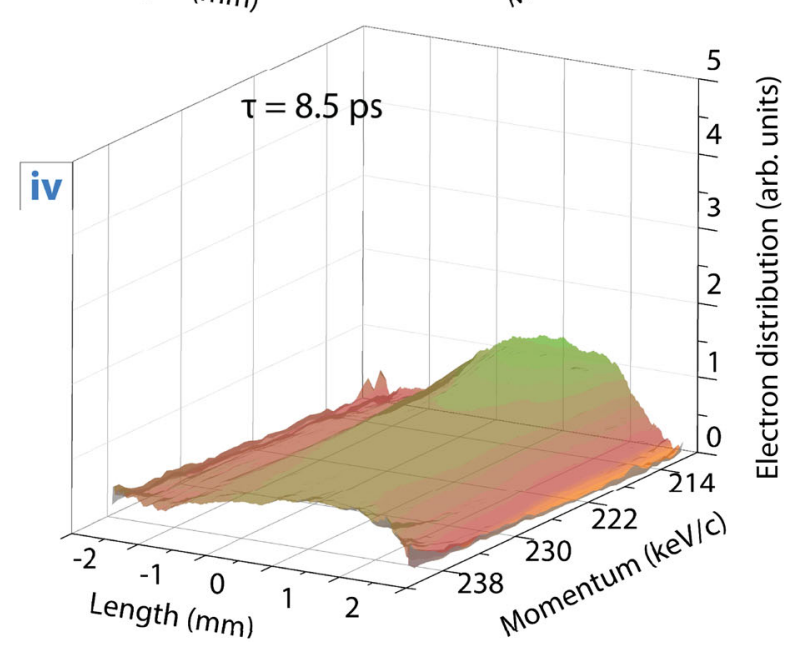

FIG. 2. Laser-electron interaction. (a) Electron-laser beam coincidence timing (blue) and normalized charge of detected accelerated electrons (red) as a function of laser-electron timing delay $(\tau)$. (b) Accelerated electron counts contained in the deflector plane at $\tau=0$. (c) Snapshots of the normalized distribution in real and momentum space of accelerated electrons at four different temporal overlaps: (i) there are no accelerated electrons when the initial electron bunch arrives at the IP 8.5 ps before the laser; (ii) distribution at the peak of total accelerated charge $(\tau=0)$ them beam is delayed by (iii) $4.25 \mathrm{ps}$ and (iv) $8.5 \mathrm{ps}$ with respect to the laser field. 

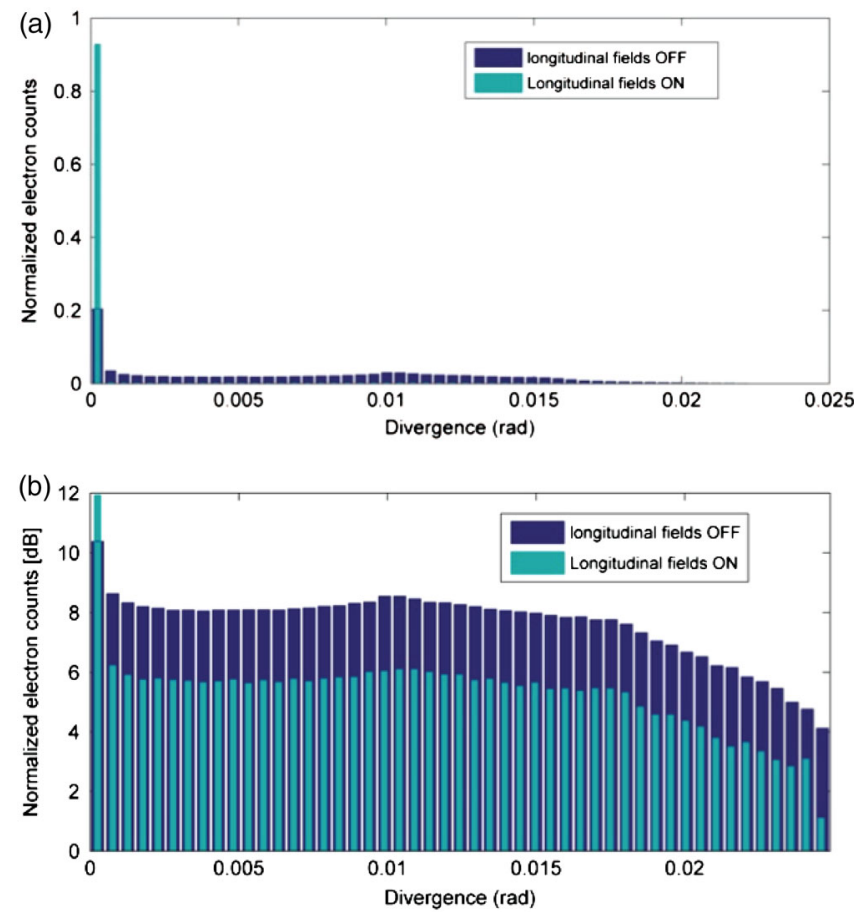

FIG. 3. Influence of longitudinal fields at focus. Simulated azimuthal distribution (divergence) of electrons above $40 \mathrm{keV}$ kinetic energy and within 25 mrad with and without the presence of longitudinal components of the driving laser field in linear (a) and logarithmic scale (b).

$3 \mathrm{kHz}$ repetition rate centered at around $800 \mathrm{~nm}$ wavelength. The routine operational peak- and average-power levels are $90 \mathrm{GW}$ and $2.4 \mathrm{~W}$, respectively. The system outputs very stable, high-quality, and nearly diffractionlimited $\mathrm{TM}_{01}$-mode beams (Sec. I in Supplementary Material [33]).

The quality of our few-cycle beams results in propagation factors close to unity and hence high focusability to access the relativistic intensity regime, defined as the region for which the ratio between the classical electron oscillation and the speed of light in vacuum-denoted as the normalized vector potential $\left(a_{0}\right)$ - exceeds unity. Here, that ratio can reach as high as $a_{0} \sim 5$. In this regime, nonrelativistic electrons can pick up a significant amount of energy from the laser field and undergo gradients in the order of several tens of $\mathrm{GeV} / \mathrm{m}$, where the maximal net electron energy gain is proportional to the field intensity as well as the beam waist at focus [14,30]. Far from the center of the beam, electrons in regions where the laser intensity is high enough will experience ponderomotive acceleration. Near the center of the beam, the nature of the laser-electron interaction is twofold: (i) nonzero azimuthal angle electric field components may offer transverse confinement of the particles while (ii) they are accelerated (and decelerated) directly from the linear force exerted by the strong longitudinal component of the electric field, uniquely available through cylindrically symmetric vector beams.

\section{EXPERIMENTAL SETUP}

Our experimental setup (Fig. 1) captures the governing physics of direct longitudinal acceleration of nonrelativistic electrons in vacuum. The $40 \mathrm{keV}$ electron beam from the photocathode is $1 \mathrm{~mm}$ long and it is assumed to exhibit an even electron distribution over all phases of the laser field. The total bunch charge thereafter is $5 \mathrm{fC}$. The laser is focused with a high-numerical aperture parabolic mirror to a waist of $1.2 \mu \mathrm{m}$ with a confocal parameter of $4 \mu \mathrm{m}$. The instrument is designed to limit the acceptance angle of electrons with less than $25 \mathrm{mrad}$ azimuthal angle. We utilize a low-energy electron spectrometer consisting of magnetic dipole deflector (tangential plane) and a microchannel plate with a nominal resolution of $100 \mathrm{eV} /$ pxl (more detail in Secs. II and III in Supplementary Material [33]).

The exact spatiotemporal coincidence of the laser and electron beam is determined by photoionization-induced spatial distortions near the interaction point (IP) using a thin carbon film aperture, represented as plasma density in Fig. 2(a). The electron undergoes observable distortions when it arrives shortly after the laser pulse at the aperture as a result of photon-induced localized charge, while no change shows if it arrives before the laser pulse. The photoemitted electron time of flight in the region of interest determines the modeled plasma density in the carbon film aperture.

We denote $\tau=0$ hereafter as to the relative time $(\tau)$ at which the accelerated charge peaks as a result of direct laser-electron interaction. Figure 2(a) shows that the time window of this interaction is $5 \mathrm{ps}$ wide, as expected from a millimeter-long electron bunch traveling at 0.39 times the speed of light. The detected charge arises from accelerated electrons only in the deflection plane and does not exhibit any symmetry with respect to the laser field vector distribution. As a result, the direct interaction manifests itself as an increased energy spread of the electron beam with very small or negligible off-axis trajectory angle. The electron bunch reveals a $5 \mathrm{~mm}$ transverse size at the detector plane and very high directionality with less than 3 mrad full width at half maximum (FWHM) off-axis divergence [Fig. 2(b)]. The relative strength of the acceleration and optimum overlap are depicted in real and momentum space at four distinct laser-electron overlapping times in Fig. 2(c). Note that the distribution only changes in strength and not in shape throughout interaction, as depicted in (ii)-(iv).

\section{LONGITUDINAL FIELD ACTION}

In order to describe this interaction, we perform simulations based on a particle-tracking model beyond the paraxial and slowly varying envelope approximations. We model pulsed radially polarized laser beams with exact, singularity-free solutions to Maxwell's equations [31,32] (Sec. IV in Supplementary Material [33]). When the laser 

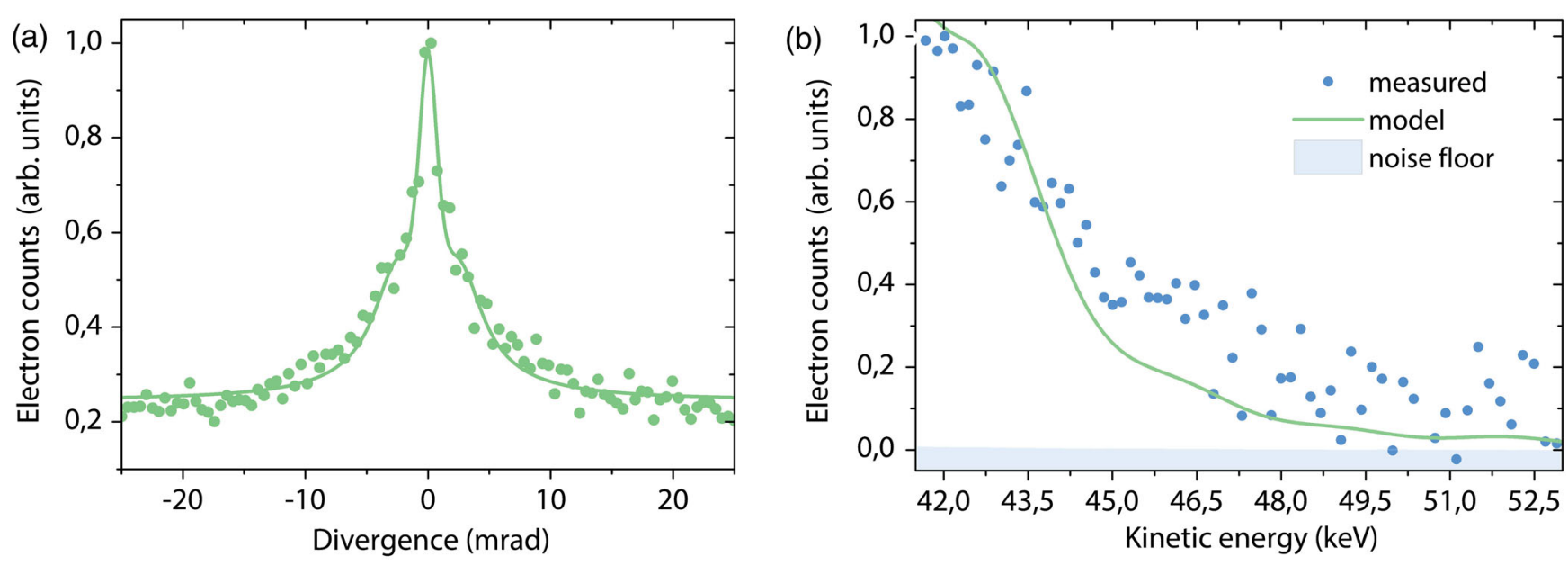

FIG. 4. Electron bunch after interaction. (a) Modeled histogram of electrons with final electron kinetic energy ranging from 42 to $53 \mathrm{keV}$ as a function of divergence with half-angle deflection of less than $25 \mathrm{mrad}$. From model, FWHM divergence of the electron bunch is expected to be $3.28 \mathrm{mrad}$. (b) Final measured and modeled kinetic energy of accelerated electron in the region of interest up to $53 \mathrm{keV}$.

pulse reaches the significantly slower $40 \mathrm{keV}$ electron bunch, the electrons contained in the interaction volume experience acceleration from both the transverse and longitudinal field components of the laser (Secs. V and VI in Supplementary Material [33]). The presence of strong longitudinal fields at the IP-that reach their maximum strength on axis - is key in enabling effective acceleration in the forward direction. We observe this effect in Fig. 3(a), which contains the modeled final azimuthal angle distribution. The predicted divergence matches the measured distribution [Fig. 2(b)] at $\tau=0$, which exhibits very small angular spread and high directionality with $3 \mathrm{mrad}$ or better divergence off axis, resulting in a very small geometric emittance of $3.6 \times 10^{-3} \mathrm{~mm}$ mrad.

The portion of electrons drawn into and accelerated in the longitudinal direction is more than 4.5 times larger in the presence of longitudinal driving fields compared to that without longitudinal field components, as shown in Fig. 3. The key element to our demonstration is the longitudinal field action and its influence in producing highly directional accelerated electrons. At the simulation level, we can quantify the influence longitudinal fields have on the amount of accelerated charge near the propagation axis (near zero azimuthal angle). We illustrate the expected divergence of the electron bunch above the initial kinetic energy and within $25 \mathrm{mrad}$ half-angle deflection angle after interaction with [Fig. 3(a)] and without [Fig. 3(b)] the presence of the longitudinal field components. For statistical purposes, the total number of particles contained in this range is virtually the same, 184968 for longitudinal fields on, and 177432 for longitudinal fields off. For comparison purposes, the distributions are normalized with respect to the total number of particles. Space charge effects are included.

The longitudinal action thus impacts unequivocally the quantity of the net accelerated charge near the optical axis as well as its emittance. Further from the beam center, a significant portion of electrons may also be launched forward in phase ranges where the longitudinal fields are accelerating and transverse fields are focusing. This combined effect may offer proper transverse confinement while accelerating particles along the beam axis. We conclude that the presence of strong longitudinal fields at focusuniquely available through relativistic cylindrical vector beams-influences and increases unambiguously the directionality of the electron bunch (improves its geometric emittance) as well as the number of electrons propelled in the forward direction.

\section{ENERGY DISTRIBUTION AFTER INTERACTION}

The measured electron kinetic energy (KE) redistribution at maximum interaction $(\tau=0)$ is shown in Fig. 4(b). The accelerated charge accounts for $4 \%$ of the total charge contained in the entire electron beam, that is, $0.2 \mathrm{fC}$ per laser pulse. The net KE energy gain is strongest within a few $\mathrm{keV}$ and is measurable and statistically reproducible to up to $12 \mathrm{keV}$. That comprises a $30 \%$ energy modulation of the initial electron $\mathrm{KE}$, which is 2 orders of magnitude higher than that achieved by current state-of-the-art dielectric accelerators [24]. As a result, this demonstrative accelerator operates at a minimum brilliance of 250 electrons/ $\left(\mathrm{s} \mathrm{mrad}^{2} \mathrm{~mm}^{2} 0.1 \% \mathrm{BW}\right)$ and a maximum on-axis gradient of $3 \mathrm{GeV} / \mathrm{m}$.

\section{CONCLUSIONS}

The key experimental result is evidence of an unprecedented laser accelerator in a purely free-space environment, where we achieve highly directional longitudinal accelerating gradients above the $\mathrm{GeV} / \mathrm{m}$ range. Moreover, this high directionality is traced back to the longitudinal 
field component from focused sub-millijoule radially polarized pulses. The performance of our demonstration is currently limited by driving laser intensity and further progress in drive source development is ongoing.

The principle is directly transferable to acceleration of charged particles to higher energies and larger brilliance by upscaling the laser intensity and wavelength, respectively. Scaling of our demonstrative concept to the petawatt-level drive laser power could lead to vacuum acceleration of electrons directly from rest to the $\mathrm{MeV}$-level [7] bunched in attosecond packets. For instance, previous work [10] suggests that a single half cycle of a multi-TW optical laser pulse could accelerate electrons from rest to around $30 \mathrm{MeV}$ with about $20 \mu \mathrm{m}$ transverse width, $2.5 \%$ energy spread, and a bunch duration below 30 as. Such performance could be instrumental in developing attosecond electron diffraction and spectroscopic techniques as well as in advancing attosecond electron injectors, among others.

Our findings leverage the scaling of usable laser power beyond material breakdown or instabilities to inspire compact high-repetition electron sources for ultrafast structural dynamics or controlled particle injection, for instance. Therefore, it sets the stage for the development of compact atomic time- and space-scale resolution electron microscopy and tomography instruments, and portable highbrilliance attosecond $\mathrm{x}$-ray sources.

\section{ACKNOWLEDGMENTS}

This work was supported by DARPA under Contract No. N66001-11-1-4192, by the Air Force Office of Scientific Research under Grant No. AFOSR-A9550-121-0499, the Center for Free-Electron Laser Science at DESY and the excellence cluster "The Hamburg Centre for Ultrafast Imaging-Structure, Dynamics and Control of Matter at the Atomic Scale" of the Deutsche Forschungsgemeinschaft. The authors kindly acknowledge William S. Graves for his support and advice with experimental implementation. L.-J. Wong acknowledges financial support from the Agency for Science, Technology and Research (A*STAR), Singapore.

S. C., L. J. W., E. N., and F.X.K. are the conceptual authors of the work presented. S. C. and E. N. carried out the experimental results and L. J. W. performed the simulations. S. C. developed the laser source. E. N., G. M., and R. J.D. M. developed the electron beam instrumentation. P. D. K. and G. L. supported with materials and instrumentation. All authors discussed the results and contributed to the final manuscript.

[1] V. Malka, J. Faure, Y. A. Gauduel, E. Lefebvre, A. Rousse, and K. T. Phuoc, Principles and applications of compact laser-plasma accelerators, Nat. Phys. 4, 447 (2008).
[2] S. P. D. Mangles et al., Monoenergetic beams of relativistic electrons from intense laser-plasma interactions, Nature (London) 431, 535 (2004).

[3] T. Toncian et al., Ultrafast laser-driven microlens to focus and energy-select mega-electron volt protons, Science 312, 410 (2006).

[4] S. Tokita, S. Inoue, S. Masuno, M. Hashida, and S. Sakabe, Single-shot ultrafast electron diffraction with a laser-accelerated sub-MeV electron pulse, Appl. Phys. Lett. 95, 111911 (2009).

[5] J. R. Dwyer, C. T. Hebeisen, R. Ernstorfer, M. Harb, V. B. Deyirmenjian, R. E. Jordan, and R. J. Dwayne Miller, Femtosecond electron diffraction: "Making the molecular movie," Phil. Trans. R. Soc. A 364, 741 (2006).

[6] S. Carbajo, E. Granados, D. Schimpf, A. Sell, K.-H. Hong, J. Moses, and F. X. Kärtner, Efficient generation of ultraintense few-cycle radially polarized laser pulses, Opt. Lett. 39, 2487 (2014).

[7] L. J. Wong and F. X. Kärtner, Direct acceleration of an electron in infinite vacuum by a pulsed radially polarized laser beam, Opt. Express 18, 25035 (2010).

[8] F. Pierre-Louis, P. Michel, and V. Charles, Direct-field electron acceleration with ultrafast radially polarized laser beams: Scaling laws and optimization, J. Phys. B 43, 025401 (2010).

[9] Y. I. Salamin, Electron acceleration from rest in vacuum by an axicon Gaussian laser beam, Phys. Rev. A 73, 043402 (2006).

[10] C. Varin and M. Piché, Relativistic attosecond electron pulses from a free-space laser-acceleration scheme, Phys. Rev. E 74, 045602 (2006).

[11] A. Sell and F. X. Kärtner, Attosecond electron bunches accelerated and compressed by radially polarized laser pulses and soft-X-ray pulses from optical undulators, J. Phys. B 47, 015601 (2014).

[12] R. Dorn, S. Quabis, and G. Leuchs, Sharper Focus for a Radially Polarized Light Beam, Phys. Rev. Lett. 91, 233901 (2003).

[13] Y. Kozawa and S. Sato, Sharper focal spot formed by higher-order radially polarized laser beams, J. Opt. Soc. 24, 1793 (2007).

[14] A. Karmakar and A. Pukhov, Collimated attosecond GeV electron bunches from ionization of high-Z material by radially polarized ultrarelativistic laser pulses, Laser Part. Beams 25, 371(2007).

[15] C. Varin, M. Piché, and M. A. Porras, Acceleration of electrons from rest to $\mathrm{GeV}$ energies by ultrashort transverse magnetic laser pulses in free space, Phys. Rev. E 71, 026603 (2005).

[16] S. Payeur, S. Fourmaux, B. E. Schmidt, J. P. MacLean, C. Tchervenkov, F. Légaré, M. Piché, and J.C. Kieffer, Generation of a beam of fast electrons by tightly focusing a radially polarized ultrashort laser pulse, Appl. Phys. Lett. 101, 041105 (2012).

[17] C. Varin et al., Direct electron acceleration with radially polarized laser beams, Appl. Sci. 3, 70 (2013).

[18] S. C. Tidwell, D. H. Ford, and W. D. Kimura, Generating radially polarized beams interferometrically, Appl. Opt. 29, 2234 (1990). 
[19] M. A. Ahmed, J. Schulz, A. Voss, O. Parriaux, J.-C. Pommier, and T. Graf, Radially polarized $3 \mathrm{~kW}$ beam from a $\mathrm{CO} 2$ laser with an intracavity resonant grating mirror, Opt. Lett. 32, 1824 (2007).

[20] R. Oron, S. Blit, N. Davidson, A. A. Friesem, Z. Bomzon, and E. Hasman, The formation of laser beams with pure azimuthal or radial polarization, Appl. Phys. Lett. 77, 3322 (2000).

[21] T. Tajima and J. M. Dawson, Laser Electron Accelerator, Phys. Rev. Lett. 43, 267 (1979).

[22] A. Buck, M. Nicolai, K. Schmid, C. M. S. Sears, A. Sävert, J. M. Mikhailova, F. Krausz, M. C. Kaluza, and L. Veisz, Real-time observation of laser-driven electron acceleration, Nat. Phys. 7, 543 (2011).

[23] W. D. Kimura, G. H. Kim, R. D. Romea, L. C. Steinhauer, I. V. Pogorelsky, K. P. Kusche, R. C. Fernow, X. Wang, and Y. Liu, Laser Acceleration of Relativistic Electrons Using the Inverse Cherenkov Effect, Phys. Rev. Lett. 74, 546 (1995).

[24] E. A. Peralta et al., Demonstration of electron acceleration in a laser-driven dielectric microstructure, Nature (London) 503, 91 (2013).

[25] A. van Steenbergen, J. Gallardo, J. Sandweiss, and J. M. Fang, Observation of Energy Gain at the BNL Inverse Free-Electron-Laser Accelerator, Phys. Rev. Lett. 77, 2690 (1996).
[26] K. Mizuno, J. Pae, T. Nozokido, and K. Furuya, Experimental evidence of the inverse Smith-Purcell effect, Nature (London) 328, 45 (1987).

[27] T. Plettner, R. L. Byer, E. Colby, B. Cowan, C. M. S. Sears, J. E. Spencer, and R. H. Siemann, Visible-Laser Acceleration of Relativistic Electrons in a Semi-Infinite Vacuum, Phys. Rev. Lett. 95, 134801 (2005).

[28] E. Esarey, C. B. Schroeder, and W. P. Leemans, Physics of laser-driven plasma-based electron accelerators, Rev. Mod. Phys. 81, 1229 (2009).

[29] C. G. R. Geddes, Cs. Toth, J. van Tilborg, E. Esarey, C. B. Schroeder, D. Bruhwiler, C. Nieter, J. Cary, and W. P. Leemans, High-quality electron beams from a laser wakefield accelerator using plasma-channel guiding, Nature (London) 431, 538 (2004).

[30] S. Carbajo, L. J. Wong, E. Nanni, D. N. Schimpf, and F. X. Kärtner, in Research in Optical Sciences (Optical Society of America, Berlin, Germany, 2014), HTu2C.6.

[31] A. April, Ultrashort, Strongly Focused Laser Pulses in Free Space (INTECH Open Access Publisher, Rijeka, Croatia, 2010).

[32] L. J. Wong, F. X. Kärtner, and S. G. Johnson, Improved beam waist formula for ultrashort, tightly focused linearly, radially, and azimuthally polarized laser pulses in free space, Opt. Lett. 39, 1258 (2014).

[33] See Supplemental Material at http://link.aps.org/ supplemental/10.1103/PhysRevAccelBeams.19.021303 for extended materials, methods, and analysis. 